TRANSACTIONS OF THE

AMERICAN MATHEMATICAL SOCIETY

Volume 217, 1976

\title{
REAL PRIME FLOWS
}

BY

H. B. KEYNES( ${ }^{1}$ ) AND D. NEWTON

ABSTRACT. In this paper, we construct examples of real-type prime flows and study these examples in detail. General properties of prime flows are studied, with emphasis on proximality conditions and properties of automorphisms. Examples of prime flows which are not POD are shown to exist, and results analogous to number-theoretic properties, such as a "unique factorisation" theorem, are shown to hold for prime flows.

0. Introduction. In this paper we continue the study of prime flows in the direction initiated in [5]. Our thrust is twofold: to enlarge the known examples of prime (actually POD) flows by exhibiting prime flows with the reals as acting group, and to generally study prime flows with regard to distinguishing various classes by dynamical properties. With regard to the examples, it is clear that the normal suspension of a discrete POD flow fails to be weakly mixing, and hence is not POD. We show that the next simplest type of example, namely a flow under a 2-valued function which is constant over two "intervals", does yield a POD flow under a mild assumption on the function values. The methods involved utilize extensions and refinements of the techniques of [5].

Our general study of prime flows was motivated by the detailed study of these examples. The study centers around proximality conditions (or, equivalently, minimal subsets of the product) and properties of the automorphisms. We first show that there are classes of prime flows which are more general than POD flows, yet enjoy many of the properties of POD flows that were developed in [5]. We call these flows purely prime, and note that they arise quite naturally by considering the flow induced by a syndetic subgroup of a POD flow. Purely prime flows are defined as a certain class of prime flows with every nontrivial automorphism being point transitive. We show the relatively surprising result that this point transitivity condition is equivalent to the fact that every other flow is either an extension or disjoint from the original flow. This enables us to obtain generalisations for purely prime flows of many of the results in [5]. In particular, we

Presented to the Society, January 23, 1975; received by the editors March 5, 1975. AMS (MOS) subject classifications (1970). Primary 54H20; Secondary 28A65.

Key words and phrases. Topological dynamics, minimal sets, seal-time flows, unique ergodicity, prime flows.

(1)Research partially supported by NSF Grant GP $32306 \mathrm{X}$.

Copyright $\odot$ 1976, American Mathematical Society 
prove "unique factorisation" of minimal $n$-fold products of purely prime flows, and show that pairwise disjointness implies minimality of the $n$-fold product. Hence, arbitrary products on distinct powers of a discrete POD flow are shown to be minimal. Finally, general properties of prime flows are studied. It is shown that the spaces are either connected or 0-dimensional (and both cases do occur). Moreover, every nontrivial automorphism of a prime flow has no equicontinuous factors and no regular factors. These observations naturally lead to the conjecture that the automorphisms themselves are prime.

1. An example of a real prime flow. First we establish some notation and describe the construction of the flow in question.

Let $\left(K, T_{\alpha}\right)$ denote the flow consisting of the unit interval $K=[0,1)$, with addition $\bmod 1$, and the rotation by $\alpha, T_{\alpha}$, with $\alpha$ irrational. Define a characteristic function $f: K \rightarrow\{0,1\}$ by $f(\gamma)=\chi_{[0, \beta]}(\gamma), \gamma \in K, \beta \in K$ and $\beta \notin Z \alpha$, and a sequence $x_{0}$ of 0 's and 1's by $x_{0}(n)=f(n \alpha), n \in Z$. Let $S=\Pi_{Z}\{0,1\}$, $\sigma$ the shift on $S$ and $X=\overline{O_{\sigma}\left(x_{0}\right)}$, the orbit closure under $\sigma$ of $x_{0} \in S$.

Proposition 1.1 [9]. $(X, \sigma)$ and $\left(K, T_{\alpha}\right)$ are minimal. There is a homomorphism $\rho:(X, \sigma) \rightarrow\left(K, T_{\alpha}\right), \rho\left(\sigma^{n} x_{0}\right)=n \alpha$, such that $\rho^{-1}(\gamma)$ is a singleton unless $\gamma \in E=(\mathrm{Z} \alpha) \cup(\beta+\mathrm{Z} \alpha)$, in which case $\rho^{-1}(\gamma)$ consists of two points. Moreover, if $\rho^{-1}(0)=\left\{x_{0}, \bar{x}_{0}\right\}$ and $\rho^{-1}(\beta)=\left\{y_{0}, \bar{y}_{0}\right\}$ with $x_{0}(0)=y_{0}(0)=1$, then $\bar{x}_{0}(0)=\bar{y}_{0}(0)=0$ and, for $n \neq 0, x_{0}(n)=\bar{x}_{0}(n)$ and $y_{0}(n)=\bar{y}_{0}(n)$.

The flow in question is constructed as a special flow over $(X, \sigma)$ under a function $g$. We define $g: X \rightarrow\left\{\beta_{1}, \beta_{2}\right\}$ by

$$
g(x)= \begin{cases}\beta_{1} & \text { if } x(0)=0, \\ \beta_{2} & \text { if } x(0)=1,\end{cases}
$$

where $\beta_{1}, \beta_{2}$ are positive real numbers, such that $\beta_{1} / \beta_{2} \notin\left\{\left(k_{1}+n \alpha\right) /\left(k_{2}+n \alpha\right) \mid\right.$ $\left.k_{1}, k_{2}, n \in \mathrm{Z}\right\}$. Set $Y^{\prime}=\{(x, t) \mid x \in X, 0 \leqslant t \leqslant g(x)\}$ and let $Y$ be the space obtained from $Y^{\prime}$ by identifying pairs $(x, g(x))$ and $(\sigma(x), 0)$. Let $\left\{S_{t}\right\}$ denote the flow on $Y$ given by

$$
S_{t}(x, s)=\left(\sigma^{n} x, s+t-g_{n}(x)\right) \text { if }-s+g_{n}(x) \leqslant t<-s+g_{n+1}(x)
$$

where for $n>0, g_{n}(x)=\Sigma_{k=0}^{n-1} g\left(\sigma^{k} x\right)$, for $n<0, g_{n}(x)=-\Sigma_{k=1}^{-n} g\left(\sigma^{-k} x\right)$ and $g_{0}(x)=0$. When convenient we will regard $X$ as a subset of $Y$ by identifying it with the set $\{(x, 0) \mid x \in X\} \subset Y$. Let $\pi: Y \rightarrow X$ be the function $\pi(x, t)=x$ $(0 \leqslant t<g(x))$.

Our main aim is to show that this flow is POD. We give the general definition of a POD transformation group in $\S 2$ (Definition 2.1). For this example we 
have to show that $\left(Y,\left\{S_{t}\right\}\right)$ is minimal and weakly mixing and that whenever $x$, $y \in Y$ with $x \neq y$ there is a $t \neq 0$ such that $S_{t} y$ is proximal to $x$.

Proposition 1.2. $\left(Y,\left\{S_{t}\right\}\right)$ is a weakly mixing minimal flow.

Proof. The minimality of any special flow over $(X, \sigma)$ follows from the minimality of $(X, \sigma)$.

Let $\lambda$ be an eigenvalue of $\left\{S_{t}\right\}$ with continuous eigenfunction $h: Y \rightarrow K$, that is,

$$
h\left(S_{t} y\right)=e^{2 \pi i \lambda t} h(y), \quad y \in Y, t \in R
$$

Let $\bar{h}$ denote the restriction of $h$ to $X$ (regarded as a subset of $Y$ ). Then $\bar{h}$ is continuous and satisfies

$$
\bar{h}(\sigma x)=e^{2 \pi i \lambda g(x)} \bar{h}(x) .
$$

Now the points $x_{0}$ and $\bar{x}_{0}$ are positively and negatively asymptotic so we can find sequences $\left(n_{k}\right),\left(m_{k}\right)$ with $n_{k}>0, n_{k} \rightarrow+\infty, m_{k}<0, m_{k} \rightarrow-\infty$ such that

$$
\lim _{k} \sigma^{n} k x_{0}=\lim _{k} \sigma^{n} k \bar{x}_{0} \text { and } \lim _{k} \sigma^{m} x_{x_{0}}=\lim _{k} \sigma^{m} k \bar{x}_{0} \text {. }
$$

We also have that $g\left(\sigma^{n} x_{0}\right)=g\left(\sigma^{n} \bar{x}_{0}\right)$ for $n \neq 0$. So for $n>0$,

$$
\begin{aligned}
\frac{\bar{h}\left(\sigma^{n} x_{0}\right)}{\bar{h}\left(x_{0}\right)} & =\frac{\bar{h}\left(\sigma^{n} x_{0}\right)}{\bar{h}\left(\sigma^{n-1} x_{0}\right)} \cdots \frac{\bar{h}\left(\sigma x_{0}\right)}{\bar{h}\left(x_{0}\right)} \\
& =\frac{\bar{h}\left(\sigma^{n} \bar{x}_{0}\right)}{\bar{h}\left(\sigma^{n-1} \bar{x}_{0}\right)} \cdots \frac{\bar{h}\left(\sigma \bar{x}_{0}\right)}{\bar{h}\left(\bar{x}_{0}\right)} \exp \left(2 \pi i \lambda\left(g\left(x_{0}\right)-g\left(\bar{x}_{0}\right)\right)\right) \\
& =\frac{\bar{h}\left(\sigma^{n} \bar{x}_{0}\right)}{\bar{h}\left(\bar{x}_{0}\right)} \cdot \exp \left(2 \pi i \lambda\left(\beta_{2}-\beta_{1}\right)\right) .
\end{aligned}
$$

Letting $n$ run through $\left\{n_{k}\right\}$ and taking limits we obtain

$$
\bar{h}\left(\bar{x}_{0}\right)=\bar{h}\left(x_{0}\right) \exp \left(2 \pi i \lambda\left(\beta_{2}-\beta_{1}\right)\right) \text {. }
$$

Similar reasoning with $n<0$ and using $\left\{m_{k}\right\}$ yields $\bar{h}\left(\bar{x}_{0}\right)=\bar{h}\left(x_{0}\right)$. Therefore $\exp \left(2 \pi i \lambda\left(\beta_{2}-\beta_{1}\right)\right)=1$, i.e. $\exp \left(2 \pi i \lambda \beta_{2}\right)=\exp \left(2 \pi i \lambda \beta_{1}\right)=\delta$ say. In other words, $\bar{h}(\sigma x)=\delta \bar{h}(x)$ and $\bar{h}\left(\sigma^{n} x_{0}\right)=\bar{h}\left(\sigma^{n} \bar{x}_{0}\right)$ for all $n$. By similar reasoning we show that $\bar{h}\left(\sigma^{n} y_{0}\right)=\bar{h}\left(\sigma^{n} \bar{y}_{0}\right)$ for all $n$. Therefore there is a continuous function $\overline{\bar{h}}$ : $K \rightarrow K$ such that $\overline{\bar{h}} \circ \rho=\bar{h}$. But then $\overline{\bar{h}}$ is a continuous eigenfunction for $T_{\alpha}$ and so $\delta=e^{2 \pi i n \alpha}$ for some $n$, i.e.

$$
\begin{array}{ll}
\lambda \beta_{1}=n \alpha+k_{1} & \text { for some } k_{1} \in \mathbf{Z}, \\
\lambda \beta_{2}=n \alpha+k_{2} & \text { for some } k_{2} \in \mathbf{Z} .
\end{array}
$$


So either $\lambda=0$ or $\beta_{1} / \beta_{2} \in\left\{\left(n \alpha+k_{1}\right) /\left(n \alpha+k_{2}\right): k_{1}, k_{2} n \in Z\right\}$. The latter possibility is excluded by the choice of $\beta_{1}$ and $\beta_{2}$ and so $\left\{S_{t}\right\}$ is weakly mixing.

Define $\tau: Y \rightarrow K$ by $\tau(y)=\rho(\pi(y))$.

The next four lemmas will show that $\left(Y,\left\{S_{t}\right\}\right)$ has the proximality property required in the definition of $P O D$.

LEMMA 1.3. Let $y_{1}, y_{2} \in Y, y_{1} \neq y_{2}$, satisfy $\tau\left(y_{1}\right)-\tau\left(y_{2}\right) \in Z a$. Then there is a $t \neq 0$ such that $S_{t} y_{1}$ is asymptotic (either positively or negatively) to $y_{2}$.

ProOF. We split the proof into a number of cases.

Case 1. $\tau\left(y_{1}\right)=\tau\left(y_{2}\right), \pi\left(y_{1}\right)=\pi\left(y_{2}\right)$.

Since $y_{1} \neq y_{2}$ this means that there are $t_{1}, t_{2}, t_{1} \neq t_{2}$ such that $S_{t_{1}} y_{1}=$ $\left(\pi\left(y_{1}\right), 0\right), S_{t_{2}} y_{2}=\left(\pi\left(y_{2}\right), 0\right)$. Then $S_{t_{1}-t_{2}} y_{1}$ is asymptotic (in fact equal) to $y_{2}$.

Case 2. $\tau\left(y_{1}\right)=\tau\left(y_{2}\right), \pi\left(y_{1}\right) \neq \pi\left(y_{2}\right)$.

In this case $\left\{\pi\left(y_{1}\right), \pi\left(y_{2}\right)\right\}=\left\{\sigma^{n} x_{0}, \sigma^{n} \bar{x}_{0}\right\}$ for some $n$ or $\left\{\pi\left(y_{1}\right), \pi\left(y_{2}\right)\right\}$ $=\left\{\sigma^{m} y_{0}, \sigma^{m} \bar{y}_{0}\right\}$ for some $m$. These are all treated in a similar way so we will suppose that $\pi\left(y_{1}\right)=\sigma^{n} x_{0}, \pi\left(y_{2}\right)=\sigma^{n} \bar{x}_{0}$. Then there are $t_{1}, t_{2}$ such that $S_{t_{1}} y_{1}=\left(\sigma^{n} x_{0}, 0\right), S_{t_{2}} y_{2}=\left(\sigma^{n} \bar{x}_{0}, 0\right)$. Using the double asymptoticity of $\sigma^{n} x_{0}$ and $\sigma^{n} \bar{x}_{0}$ under $\sigma$ and the fact that $g\left(\sigma^{m} x_{0}\right)=g\left(\sigma^{m} \bar{x}_{0}\right)$ for $m \neq 0$ we can assert that $S_{t_{1}-t_{2}} y_{1}$ is asymptotic to $y_{2}$ (negatively if $n<0$, positively if $n>0$ ). We can, by similar reasoning, assert that $S_{t_{1}-t_{2}+\beta_{1}-\beta_{2}} y_{1}$ is asymptotic to $y_{2}$ (positively if $n<0$, negatively if $n>0$ ). Note that as we 'pass through' the points $\left(x_{0}, 0\right),\left(\bar{x}_{0}, 0\right)$ we have $g\left(\bar{x}_{0}\right)-g\left(x_{0}\right)=\beta_{1}-\beta_{2}$. Since one of the numbers $t_{1}-t_{2}, t_{1}-t_{2}+\beta_{1}-\beta_{2}$ must be nonzero, we have the lemma in Case 2 .

Case 3. $\tau\left(y_{1}\right)-\tau\left(y_{2}\right)=k \alpha, k \neq 0$.

Then, without loss of generality, $T_{\alpha}^{-k} \tau\left(y_{1}\right)=\tau\left(y_{2}\right)$ for some $k>0$, that is $\rho\left(\sigma^{-k} \pi\left(y_{1}\right)\right)=\rho\left(\pi\left(y_{2}\right)\right)$ so for some $t<0, \rho \pi\left(S_{t} y_{1}\right)=\rho \pi\left(y_{2}\right)$. So either: $\pi\left(S_{t} y_{1}\right)=\pi\left(y_{2}\right)$ in which case for some $t_{1}<-t$ we have that $S_{t_{1}+t} y_{1}$ is asymptotic (in fact equal) to $y_{2}$, or: $\left\{\pi\left(S_{t} y_{1}\right), \pi\left(y_{2}\right)\right\}=\left\{\sigma^{n} x_{0}, \sigma^{n} \bar{x}_{0}\right\}$ or $\left\{\pi\left(S_{t} y_{1}\right), \pi\left(y_{2}\right)\right\}=\left\{\sigma^{m} y_{0}, \sigma^{m} \bar{y}_{0}\right\}$. In this case there are two values of $\tau$ for which $S_{\tau+t} y_{1}$ is asymptotic to $y_{2}$. Choosing one value of $\tau+t$ which is not zero we have the lemma for Case 3.

We have shown the proximality property for those pairs of points $y_{1}, y_{2}$ for which $\tau\left(y_{1}\right)-\tau\left(y_{2}\right) \in Z \alpha$. We now go on to consider the pairs for which this is not the case.

Define functions $n: X \times R \rightarrow Z$ and $r: X \times R \rightarrow\left[0, \max \left(\beta_{1}, \beta_{2}\right)\right)$ by the equation

$$
S_{t}(x, 0)=\left(\sigma^{n(x, t)} x, r(x, t)\right)
$$


These functions are, of course, not continuous. However $n(x, t)$ is onto and nondecreasing in $t$.

For a fixed pair of points $x, y \in X^{\prime}$ we consider the difference $n(x, t)-$ $n(y, t)$.

LEMMA 1.4. $n(x, t)-n(y, t)$ is unbounded as a function of $t$ if $\rho(x)-\rho(y) \notin Z \alpha$.

Proof. Assume that $n(x, t)-n(y, t)$ is bounded. By definition

$$
t=\sum_{i=0}^{n(x, t)-1} g\left(\sigma^{i} x\right)+r(x, t), \quad t=\sum_{i=0}^{n(y, t)-1} g\left(\sigma^{i} y\right)+r(y, t) .
$$

(We are assuming $t>0$ here.) So

$$
\sum_{i=0}^{n(x, t)-1}\left(g\left(\sigma^{i} x\right)-g\left(\sigma^{i} y\right)\right)= \begin{cases}r(y, t)-r(x, t)+\sum_{t=n(x, t)}^{n(y, t)-1} g\left(\sigma^{i} y\right) & \text { if } n(y, t)>n(x, t), \\ r(y, t)-r(x, t) & \text { if } n(y, t)=n(x, t), \\ r(y, t)-r(x, t)-\sum_{i=n(y, t)}^{n(x, t)-1} g\left(\sigma^{i} y\right) & \text { if } n(y, t)<n(x, t) .\end{cases}
$$

But if $|n(x, t)-n(y, t)|<M$ then the right-hand side of the above expression is bounded above by $(M+1) \max \left\{\beta_{1}, \beta_{2}\right\}$ and below by $-(M+1) \max \left\{\beta_{1}, \beta_{2}\right\}$. Since $n(x, t)$ is onto $\mathrm{Z}^{+}$for $t>0$ we have that $\sum_{i=0}^{n-1}\left(g\left(\sigma^{i} x\right)-g\left(\sigma^{i} y\right)\right)$ is bounded for all $n>0$.

However, denoting $\chi_{\{x(0)=0\}}$ by $\chi_{0}$ and $\chi_{\{x(0)=1\}}$ by $\chi_{1}$, we have that $g=$ $\beta_{1} \chi_{1}+\beta_{2} \chi_{0}=\left(\beta_{1}-\beta_{2}\right) \chi_{1}+\beta_{2}$. So

$$
\sum_{i=0}^{n-1}\left(g\left(\sigma^{i} x\right)-g\left(\sigma^{i} y\right)\right)=\left(\beta_{1}-\beta_{2}\right) \sum_{i=0}^{n-1}\left(\chi_{1}\left(\sigma^{i} x\right)-\chi_{1}\left(\sigma^{i} y\right)\right) .
$$

Now we are assuming $\rho(x)-\rho(y) \notin Z \alpha$. If neither $\rho(x)$ or $\rho(y) \in(\mathrm{Z} \alpha) \cup$ $(\beta+Z \alpha)$, then we have

$$
\begin{aligned}
& \sum_{i=0}^{n-1}\left(g\left(\sigma^{i} x\right)-g\left(\sigma^{i} y\right)\right) \\
& \quad=\left(\beta_{1}-\beta_{2}\right) \sum_{i=0}^{n-1} \chi_{[0, \beta)}(\rho(x)+i \alpha)-\chi_{[0, \beta)}(\rho(y)+i \alpha) .
\end{aligned}
$$

If either $\rho(x)$ or $\rho(y) \in(Z \alpha) \cup(\beta+Z \alpha)$, then we may eventually have either

$$
\left|\sum_{i=0}^{n-1} \chi_{1}\left(\sigma^{i} x\right)-\chi_{[0, \beta)}(\rho(x)+i \alpha)\right|=1
$$

or 


$$
\left|\sum_{i=0}^{n-1} \chi_{1}\left(\sigma^{i} y\right)-\chi_{[0, \beta)}(\rho(y)+i \alpha)\right|=1
$$

and $(*)$ holds up to a constant. But the right-hand side of $(*)$ is bounded only if $\rho(x)-\rho(y) \in Z \alpha$ [5, Corollary 2.3]. So in either case, we have contradiction, and the lemma is proved.

Lemma 1.5. Let $y_{1}, y_{2} \in Y$ with $\tau\left(y_{1}\right)-\tau\left(y_{2}\right) \notin \mathrm{Z} \alpha$. Then there is a $t_{0}$ and $\left(\bar{y}_{1}, \bar{y}_{2}\right) \in \overline{O\left(S_{t_{0}} y_{1}, y_{2}\right)}$ with $\tau\left(\bar{y}_{1}\right)-\tau\left(\bar{y}_{2}\right) \in\{w, w+\alpha, w-\alpha\}$, for any $w \in K$.

PROOF. Let $y_{1}=\left(\pi\left(y_{1}\right), t_{1}\right), y_{2}=\left(\pi\left(y_{2}\right), t_{2}\right)$ and put $t_{0}=t_{2}-t_{1}$.

Then, for $t>0$,

$$
\tau\left(S_{t+t_{0}} y_{1}\right)=\tau\left(y_{1}\right)+n\left(\pi\left(y_{1}\right), t+t_{2}\right) \alpha, \quad \tau\left(S_{t} y_{2}\right)=\tau\left(y_{2}\right)+n\left(\pi\left(y_{2}\right), t+t_{2}\right) \alpha \text {. }
$$

So

$$
\tau\left(S_{t+t_{0}} y_{1}\right)-\tau\left(S_{t} y_{2}\right)=r\left(y_{1}\right)-\tau\left(y_{2}\right)+\left(n\left(\pi\left(y_{1}\right), t+t_{2}\right)-n\left(\pi\left(y_{2}\right), t+t_{2}\right)\right) \alpha .
$$

Since $n\left(\pi\left(y_{1}\right), t+t_{2}\right)-n\left(\pi\left(y_{2}\right), t+t_{2}\right)$ is unbounded as a function of $f$ and changes in increments of \pm 1 , then its range contains either $Z^{+}$or $\mathbf{Z}^{-}$. Therefore for any $w \in K$ we can find a sequence $\left(t_{n}\right)$ for which $\tau\left(S_{t_{n}+t_{0}} y_{1}\right)-\tau\left(S_{t_{n}} y_{2}\right)$ $\rightarrow w$. We now choose a limit point $\left(\bar{y}_{1}, \bar{y}_{2}\right)$ of the sequence $\left(S_{t_{n}+t_{0}} y, S_{t_{n}} y_{2}\right)$ and the lemma is proved. (Note that if $z_{n} \rightarrow z, \tau\left(z_{n}\right) \rightarrow x$, then $\tau(z)=x$ or $x+\alpha$.)

REMARK 1.6. In Lemma 1.5 it is clear that we can choose $t_{0}$ to satisfy $\left|t_{0}\right|<\max \left\{\beta_{1}, \beta_{2}\right\}$. For our next lemma we will need a lower bound on the $t$ involved in Case 3 of the proof of Lemma 1.3, that is, if $\tau\left(y_{1}\right)-\tau\left(y_{2}\right)=k \alpha, k$ $\neq 0$, then there is a $t \neq 0$ such that $S_{t} y_{1}$ is asymptotic to $y_{2}$. A careful reading of the proof shows that as $|k| \rightarrow \infty$ so also does $|t| \rightarrow \infty$. Thus there is $K$ such that $|k|>K$ implies $|t|>\max \left\{\beta_{1}, \beta_{2}\right\}$.

Proposition 1.7. If $\tau\left(y_{1}\right)-\tau\left(y_{2}\right) \notin Z \alpha$, then for some $t \neq 0, S_{t} y_{1}$ is proximal to $y_{2}$.

Proof. By Lemma 1.5 there is a $t_{0},\left|t_{0}\right|<\max \left\{\beta_{1}, \beta_{2}\right\}$, and $\bar{y}_{1}, \bar{y}_{2} \in$ $\left.\overline{O\left(S_{t_{0}} y_{1}, y_{2}\right.}\right)$ with $\tau\left(y_{1}\right)-\tau\left(y_{2}\right)=k \alpha$ where $k$ is chosen to satisfy $|k|>K$ (see Remark 1.7). Now, by Lemma 1.3 , there is a $t$ such that $S_{t} \bar{y}_{1}$ is proximal to $\bar{y}_{2}$ and, by our choice of $k,|t|>\max \left\{\beta_{1}, \beta_{2}\right\}>\left|t_{0}\right|$. But then $S_{t+t_{0}} y_{1}$ is proximal to $y_{2}$ and $\left|t+t_{0}\right|>0$. The proof is complete.

Proposition 1.7 together with Lemma 1.3 and Proposition 1.2 shows that $\left(Y,\left\{S_{t}\right\}\right)$ is a POD flow. 
THEOREM 1.8. A real POD flow is prime. Moreover for each $t \neq 0$, the discrete flow $\left(Y, S_{t}\right)$ is prime but not $P O D$.

Proof. These results will follow from general results in $\$ 2$ (see Propositions 2.2 and 2.8).

Finally we note that our example is uniquely ergodic. This follows from the fact that a special flow over a uniquely ergodic flow is uniquely ergodic. In fact let $(X, \sigma)$ be uniquely ergodic with invariant measure $\mu$ and let $\left(Y,\left\{S_{t}\right\}\right)$ be the special flow over $(X, \sigma)$ under a continuous function $g$. Define an invariant measure $\bar{\mu}$ on $Y$ by

$$
\int f d \bar{\mu}=\int_{X} \int_{0}^{g(x)} f\left(S_{t} x\right) d t d \mu / \int_{X} g(x) d \mu \quad(f \in C(Y)) .
$$

For $f \in C(Y)$ define $\bar{f} \in C(X)$ by $\bar{f}(x)=\int_{0}^{g(x)} f\left(S_{t} x\right) d t$. Now fix $y \in Y$, let $x=\pi y, y=S_{\tau} x, 0 \leqslant \tau<g(x)$. For any $T>0$ we can write $T=g(x)+\cdots+$ $g\left(\sigma^{n} x\right)+\tau_{T}, 0 \leqslant \tau_{T}<g\left(\sigma^{n+1} x\right)$. Then

$$
\begin{aligned}
\frac{1}{T} \int_{0}^{T} f\left(S_{t} y\right) d t= & \frac{1}{T}\left[\int_{0}^{T} f\left(S_{t} x\right) d t-\int_{0}^{T} f\left(S_{t} x\right) d t+\int_{T}^{T+\tau} f\left(S_{t} x\right) d t\right] \\
= & \frac{1}{T}\left[\sum_{i=0}^{n} \bar{f}\left(\sigma^{i} x\right)-\int_{0}^{\tau} f\left(S_{t} x\right) d t+\int_{T-\tau}^{T+\tau} f\left(S_{t} x\right) d t\right] \\
= & \frac{n+1}{\sum_{i=0}^{n} g\left(\sigma^{i} x\right)} \cdot \frac{\sum_{i=0}^{n} g\left(\sigma^{i} x\right)}{T} \cdot \frac{1}{n+1} \sum_{i=0}^{n} \bar{f}\left(\sigma^{i} x\right) \\
& +\frac{1}{T}\left[\int_{T-\tau}^{T+\tau} f\left(S_{t} x\right) d t-\int_{0}^{\tau} f\left(S_{t} x\right) d t\right]
\end{aligned}
$$

Letting $T \rightarrow \infty$ and using unique ergodicity of $\sigma$ we get

$$
\lim _{T \rightarrow \infty} \frac{1}{T} \int_{0}^{T} f\left(S_{t} y\right) d t=\frac{1}{\int g d \mu} \cdot \int \bar{f} d \mu=\int f d \bar{\mu} .
$$

So it follows that $\left(Y,\left\{S_{t}\right\}\right)$ is uniquely ergodic with invariant measure $\bar{\mu}$.

2. Classes of prime flows and their properties. In this section we will study several distinct types of minimal prime flows. Recall that a flow is prime if every factor which is not the 1-point flow is isomorphic via the given homomorphism to the original flow. This is equivalent to asserting that if $R$ is an invariant closed equivalence relation (ICER) on $X$, then either $R=X \times X$ or $R=$ $\Delta$ (the diagonal of $X \times X$ ). With this definition, we ignore any group topologies on the acting group and thus, from the viewpoint of factors, regard the group as 
discrete. Also it immediately follows that every prime flow is coalescent. In general the categories of prime flows that we consider will be distinguished by disjointness relations and various roles of the group of automorphisms of the flow involved.

Throughout this section $(X, T)$ will denote an arbitrary, not necessarily minimal, transformation group, with $X$ being a compact Hausdorff space and $T$ an abelian group. The words "flow" and "transformation group" will be used interchangeably.

Definition 2.1. $(X, T)$ is a POD transformation group if:

(i) for every $t \neq e$, the discrete flow $\left(X, \pi^{t}\right)$ is minimal,

(ii) if $x, y \in X, x \neq y$, then the orbit closure $\overline{O(x, y)} \supset \Gamma_{t}$ for some $t \neq$ $e$, where $\Gamma_{t}=\{(z, z t): z \in X\}$ is the graph of $\pi^{t}$.

Note that, since $(X, T)$ is minimal, (ii) is equivalent to $x$ and $y t^{-1}$ being proximal. In the case of a real flow condition (i) is implied by weakly mixing and minimality. Thus we see that the example in $\$ 1$ is a POD flow in this sense.

Many of the properties of POD integer flows, discussed in [5], easily carry over. For example we have

Proposition 2.2. If $(X, T)$ is $P O D$ then $(X, T)$ is prime.

Proof. Let $R$ be an ICER on $X$ with $R \neq \Delta$. Choose a point $(x, y) \in R$ with $x \neq y$. Then $R \supset \overline{O(x, y)} \supset \Gamma_{t}$ for some $t \neq e$. Then $R \supset\left\{\left(w, w t^{n}\right): w \in\right.$ $X, n \in \mathrm{Z}$ \} and so by the minimality of $\left(X, \pi^{t}\right), R=X \times X$, and the proposition is proved.

We shall now study several classes of flows which lie between POD and minimal prime. First we give several definitions. We denote the automorphism group of $(X, T)$ by $\operatorname{Aut}(X, T)$. Notice that the assumption that $T$ is abelian implies that each $\pi^{t} \in \operatorname{Aut}(X, T)$.

Definition 2.3. A transformation group $(X, T)$ is

(i) type $I$ if the smallest closed equivalence relation containing the orbit relation is $X \times X$,

(ii) type II if, for every $x \neq y$, there exists $\phi \in \operatorname{Aut}(X, T)$ with $\phi \neq \mathrm{id}$ and $\phi^{-1}(x)$ proximal to $y$,

(iii) type III if for every $\phi \in \operatorname{Aut}(X, T)$ with $\phi \neq$ id, the discrete flow $(X$, $\phi)$ is type I.

Note that if we are discussing an integer flow $(Z, \psi)$ then we can replace the orbit relation in (i) by the graph $\Gamma_{\psi}$. Also if $(X, T)$ is minimal, condition (ii) is equivalent to $\overline{O(x, y)} \supset \Gamma_{\phi}$.

Proposition 2.4. (i) If $(X, T)$ is prime then it is type III.

(ii) If $(X, T)$ is minimal and types II and III then it is prime. Thus, a type II minimal flow is prime iff it is type III. 
Proof. (i) Let $\phi \in \operatorname{Aut}(X, T), \phi \neq$ id, and let $R$ be a closed equivalence relation with $R \supset \Gamma_{\phi}$. Since $\Gamma_{\phi} t=\Gamma_{\phi}(t \in T)$, then $\Gamma_{\phi} \subset \bigcap_{t \in T} R t=R_{0}$. Since $R_{0}$ is an ICER and $R_{0} \neq \Delta$, then $R_{0}=X \times X$ by the fact that $(X, T)$ is prime. Hence $R=X \times X$ and we get the result.

(ii) Let $R$ be an ICER with $R \neq \Delta$. Choose a point $(x, y) \in R$ with $x \neq$ y. Then $\overline{O(x, y)} \supset \Gamma_{\phi}$ for some $\phi \in \operatorname{Aut}(X, T)$ with $\phi \neq \mathrm{id}$, and so $\Gamma_{\phi} \subset R$. Since $(X, T)$ is type III it follows that $R=X \times X$, completing the proof.

Later we will give an example of a minimal type III integer flow which is not prime.

If $(X, T)$ is POD, then it is both types II and III. This follows directly from the fact that $\operatorname{Aut}(X, T)=\left\{\pi^{t}: t \in T\right\}$. For if $\phi:(X, T) \rightarrow(X, T)$ is an endomorphism them $\Gamma_{\phi}$ is a minimal subset of $(X \times X, T)$ and so $\Gamma_{\phi}=\Gamma_{t}$ for some $t$. Hence $\phi=\pi^{t}$ as required. A minimal type II transformation group is both regular [1] and coalescent. So we see that, as in the integer case, a POD flow is regular.

The above observation shows that one advantage that a POD transformation group has over a minimal transformation group of types II and III is that the type III condition is satisfied in the most obvious way, namely by minimality of the nontrivial automorphisms. The next few results show that if we hypothesize a weaker property (point transitivity) which still yields the type III property, then we can recover many of the properties that POD transformation groups enjoy.

Definition 2.5. A minimal transformation group $(X, T)$ is called purely prime if (i) it is type II, (ii) if $\phi \in \operatorname{Aut}(X, T)$ with $\phi \neq \mathrm{id}$, then $(X, \phi)$ is pointtransitive.

It is clear that $\mathrm{POD} \Rightarrow$ purely prime $\Rightarrow$ prime. Below in Proposition 2.8, we will show that examples of purely prime, non-POD, transformation groups arise naturally.

Proposition 2.6. Let $(X, T)$ be purely prime. If $(Z, T)$ is minimal and not an extension of $(X, T)$, then $(X, T)$ and $(Z, T)$ are disjoint.

Proof. The argument follows the lines of [5, Theorem 4.3]. Let $M$ be a minimal subset in $(X \times Z, T)$. Since $(Z, T)$ is not an extension of $(X, T)$ we can choose $x, y \in X, z \in Z$ with $x \neq y$ and $(x, z),(y, z) \in M$. Now $\overline{O(x, y)} \supset \Gamma_{\phi}$, for some $\phi \in \operatorname{Aut}(X, T), \phi \neq \mathrm{id}$. Since $(X, \phi)$ is point transitive, there is $w \in X$ with $\overline{O_{\phi}(w)}=X$. If $(x, y) t_{n} \rightarrow(w, \phi(w))$ and $z_{0}$ is a cluster point of $\left(z t_{n}\right)$, then $\left(w, z_{0}\right),\left(\phi(w), z_{0}\right) \in M$. Hence $\phi \times \operatorname{id}(M) \cap M \neq \varnothing$ and so $\phi^{n} \times \operatorname{id}(M)=M$ for every $n$. Therefore $O_{\phi}(w) \times\left\{z_{0}\right\} \subset M$ and so $X \times\left\{z_{0}\right\} \in M$. The minimality of $(Z, T)$ now implies $M=X \times Z$ and so $(X, T)$ and $(Z, T)$ are disjoint.

Corollary 2.7. Let $(X, T)$ be purely prime and $(Z, T)$ minimal. Then: 
(i) $(X, T)$ and $(Z, T)$ are disjoint if and only if their only common factor is the trivial flow.

(ii) If $(Z, T)$ is also prime, then $(X, T)$ and $(Z, T)$ are either isomorphic or disjoint.

Recall that $S$ being syndetic in $T$ means $S K=T$ for some compact subset $K$ of $T$.

Proposition 2.8. Let $(X, T)$ be POD and $S$ a nontrivial syndetic subgroup of T. Then $(X, S)$ is purely prime and not POD.

Proof. We will first show that $\operatorname{Aut}(X, S)=\left\{\pi^{t}: t \in T\right\}$. Let $W$ be a minimal subset of $(X \times X, S)$ and choose $K$ compact with $S K=T$. Then $W K$ is a closed $T$-invariant subset of $X \times X$. Thus there is a minimal set $M$ of $(X \times X$, $T)$ such that $M \subset W K$. Since $(X, T)$ is POD, $M=\Gamma_{t}$ for some $t$. Pick $x \in X$ and $k^{-1} \in K$ with $(x, x t) \in W k^{-1}$ and $(x k, x k t) \in W$. Since $(X, S)$ is minimal, $\Gamma_{t} \subset W$, and since $\Gamma_{t}$ is minimal in $(X \times X, S), W=\Gamma_{t}$. Now if $\phi \in \operatorname{Aut}(X, S)$, then $\Gamma_{\phi}$ is minimal in $(X \times X, S)$. Hence $\Gamma_{\phi}=\Gamma_{t}$ for some $t$ and so $\phi=\pi^{t}$. This shows that $(X, S)$ has the point transitivity property of its nonidentity automorphisms. Finally we show that $(X, S)$ is type II. Let $x, y \in X, x \neq y$. Then $\overline{O_{T}(x, y)}=\overline{O_{S}(x, y) K}$. By the POD property of $(X, T), \overline{O_{T}(x, y)} \supset \Gamma_{t}$ for some $t \neq e$ and so by the first part of this proof, replacing $W$ by $\overline{O_{S}(x, y)}$ we get $\Gamma_{t}$ $\subset \overline{O_{S}(x, y)}$. Therefore $(X, S)$ is purely prime.

To show that $(X, S)$ is not POD we note that if it were then we would have $\left\{\pi^{s}: s \in S\right\}=\left\{\pi^{t}: t \in T\right\}$. However, $(X, T)$ is freely acting since $\left(X, \pi^{t}\right)$ is minimal $(t \in T)$. So this is not possible and $(X, S)$ is not POD.

This proposition yields that for a discrete POD flow $(X, \phi),\left(X, \phi^{p}\right), p \neq$ \pm 1 , is purely prime and not POD, and for a real POD flow $\left(Y,\left\{S_{t}\right\}\right)$, each discrete flow $\left(Y, S_{t}\right), t \neq 0$, is purely prime and not POD. This observation together with Proposition 2.6 will be heavily used in proving theoretic analogues for these flows, including a substantial generalisation of [5, Theorem 4.8].

One might wonder if any other weaker property on a prime transformation group would yield Proposition 2.6 and other properties similar to POD transformation groups. The next proposition somewhat surprisingly shows that for a prime transformation group to satisfy Proposition 2.6 we must have the condition of point-transitivity on the nontrivial automorphisms. Thus, for a type II minimal prime flow, pure primeness is equivalent to the disjointness-of-nonextensions property.

We shall use the algebraic theory of minimal sets as developed in [2] and [4] to prove the above result. Thus, our minimal prime flow $(X, T)$ will be represented by $X=\mid 01$, where $0 \subset C(M), M$ the distinguished minimal right ideal 
in $\beta T$. Following the notation of [2], we shall work with the topology $T$ induced on $M$ via the canonical map $\pi:(M, T) \rightarrow(|O|, T)$. Sets of the form $h(W)=$ $\{q: q \in M, W \in q\}$, where $h(W)$ is an 0 -neighbourhood of $p \in M$, are noted there to be basic $T$-neighbourhoods of $p$. Also recalling that $O$ induces a topology $\tau(0)$ on $G$, we have that if $\alpha \in G,(U, N)=\{\beta: \beta \in G$ and $U \beta \cap N \neq \phi\}$, where $h(U)$ is an 0 -neighbourhood of $\alpha$ and $N \in u$, is $\tau(0)$-open. We first note that the restriction on $N$ is unnecessary.

LEMma 2.9. Let $T$ be abelian, $h(U)$ a nonempty 0 -neighbourhood, and $V \subset T$ with $h(V) \cap M \neq \varnothing$. Then $(U, V)$ has a nonempty $\tau(O)$-interior.

Proof. Since $G$ is T-dense, there exists $\alpha \in$ int $_{T} h(U)$ and so $h(U)$ is an 0 -neighbourhood of $\alpha$. We now follow the proof of $[2,11.14 .1]$. Choose $f$ and $\Delta$ as in this proof, and $\beta \in G$ with $\beta \in h(V)$. Setting $K=G-(U, V)$ we have that $\left\langle f^{K}, t\right\rangle=0(t \in V)$. Hence $\left\langle f^{K}, \beta\right\rangle=0$. Now if $\gamma \in \operatorname{cls}_{\tau(0)}(K \beta)=$ $\left(\operatorname{cls}_{\tau(0)} K\right) \beta$, then $\gamma \beta^{-1} \in \operatorname{cls}_{\tau(0)} K$ and thus $f \gamma \beta^{-1} \leqslant f^{K} u, f \gamma \leqslant f^{K} u \beta=f^{K} \beta$. Hence $\langle f, \gamma\rangle=\langle f \gamma, e\rangle \leqslant\left\langle f^{K}, \beta\right\rangle=0$, and so $\gamma \notin \Delta$. This shows that $\Delta C$ $\left(\operatorname{cls}_{\tau(0)} K\right)^{\prime} \beta$, whence $\Delta \beta^{-1} \subset\left(\operatorname{cls}_{\tau(0)} K\right)^{\prime}=\operatorname{int}_{\tau(0)}(U, V)$. This completes the proof.

We now proceed to the proof of the desired result. We let $A$ be the group of 0 . Recall $[2,11.18,11.19]$ that every automorphism of $(|O|, T)$ is associated (up to $A$ ) with some $\alpha \in G$.

Proposition 2.10. Let $(X, T)=(101, T)$ be minimal prime, $X$ metric, and satisfy the condition: if $(Z, T)$ is minimal and $(Z, T) \underset{\mp}{\mp}(X, T)$, then $(X, T)$ and $(Z, T)$ are disjoint. Let $\phi \in \operatorname{Aut}(X, T)$ with $\phi \neq \mathrm{id}$. Then $(X, \phi)$ is point transitive.

Proof. Pick $\alpha \in G$ such that $\alpha$ represents $\phi$. Then $\phi \neq$ id implies $\alpha \notin A$. Let $L$ be the subgroup of $G$ generated by $\alpha$, and $K=\operatorname{cls}_{\tau} L$. Then $K$ is a $\tau$ closed subgroup. We claim that $A K=G$. Otherwise $A K \varsubsetneqq G$, and the flow $(|O(K)|, T)$ is not disjoint from $(|O|, T)[2,18.7]$. But then $(|O(K)|, T) \rightarrow$ $(|0|, T)$ and so $K \subset A$. This is impossible, since $\alpha \notin A$. Since $\operatorname{cls}_{\tau(0)} L=A K$ by [3, Proposition 3.2], then $\operatorname{cls}_{\tau(0)} L=G$. Finally, let $h(U)$ and $h(V)$ be any $O$-neighbourhoods. By 2.9, $(U, V)$ has a nonempty $\tau(O)$-interior, and so $L \cap$ $(U, V) \neq \varnothing$. If $\alpha^{n} \in(U, V)$, then $U \alpha^{n} \cap V \neq \varnothing$. This means that $\alpha^{n} t \in h(U)$ for some $t \in V$, and hence $\alpha^{n} h(V) \cap h(U) \neq \varnothing$. Since $(X, \phi)$ is represented by $(M, \alpha)$ with the topology $T$, and $X$ is metric, it follows that $(X, \phi)$ is point transitive. holds.

We shall shortly see in Proposition 2.11 that the metric assumption usually

On the basis of the examples we have examined, we make the following conjecture. 
Conjecture A. If $(X, T)$ is purely prime, and if $\phi \in \operatorname{Aut}(X, T), \phi \neq \mathrm{id}$, then $(X, \phi)$ is minimal.

We now return to the general consequences of primeness. The first result concerns the topology of $X$.

Proposition 2.11. Let $(X, T)$ be minimal prime. Then:

(i) If $T$ is $\sigma$-compact, then $X$ is metric.

(ii) $X$ is either connected or 0-dimensional. Moreover, if $X$ is 0-dimensional and $T$ is countable, then $(X, T)$ is expansive.

Proof. (i) Let $f \in C(X)$. Then $\langle f\rangle=C(X)$, where $\langle f\rangle$ denotes the least closed invariant subalgebra containing $f$. Now $\langle f\rangle$ is separable [6, Lemma 2.1], proving this assertion.

(ii) Suppose $X$ is not connected. Then $X=B_{0} \cup B_{1}$, where $B_{0}$ and $B_{1}$ are nonempty closed-open subsets of $X$. Let $\phi=\chi_{B_{0}}$ and define $\pi: X \rightarrow$ $\{0,1\}^{T}$ by $\pi(x)=(\phi(x t) \mid t \in T)$. Considering the transformation group $\left.(\{0,1\}\}^{T}, T\right)$ with $\left(x_{t} \mid t \in T\right) s=\left(x_{t s} \mid t \in T\right)$ (taking the product topology on $\{0,1\}^{T}$ and ignoring any topology on $\left.T\right)$, it is easily seen that $\pi$ is a homomorphism. Since $\pi(X)$ is nontrivial then $\pi$ is an isomorphism of $(X, T)$ onto $(\pi(X)$, T). Thus $X$ is 0 -dimensional. Finally, if $T$ is countable, $\left(\{0,1\}^{T}, T\right)$ is expansive. Thus $(X, T)$ is expansive and the proof is complete.

Note that in the case of $T$ being the integers that both the connected and 0 -dimensional cases do occur.

We now consider the dynamical properties of the automorphisms of prime flows.

Lemma 2.12. Let $(X, T)$ be minimal prime and $\phi \in \operatorname{Aut}(X, T)$ with $\phi \neq$ id. Suppose $\pi:(X, \phi) \stackrel{\rightarrow}{\rightarrow}(W, \psi)$, with $W$ nontrivial. Then $(X, \phi)$ is isomorphic to a subflow of the flow $\left(W^{T}, \hat{\psi}\right)$ with action $\hat{\psi}\left(w_{t} \mid t \in T\right)=\left(\psi\left(w_{t}\right) \mid t \in T\right)$.

Proof. Define $\rho: X \rightarrow W^{T}$ by $\rho(x)=(\pi(x t) \mid t \in T)$. Then clearly $\rho:$ $(X, T) \widetilde{\rightrightarrows}\left(W^{T}, T\right)$ is a homomorphism into. Since $\rho(X)$ is nontrivial, then $\rho$ is an isomorphism and $\rho(X)$ is homeomorphic to $X$. Moreover, $\rho(\phi(x))=$ $(\pi(\phi(x) t) \mid t \in T)=(\pi(\phi(x t)) \mid t \in T)=(\psi(\pi(x t)) \mid t \in T)=\hat{\psi}(\rho(x))$, thus proving the lemma.

Proposition 2.13. Let $(X, T)$ be minimal prime with $X$ infinite and $\phi \in$ $\operatorname{Aut}(X, T)$ with $\phi \neq \mathrm{id}$. Then $(X, \phi)$ has no nontrivial equicontinuous factors.

Proof. Suppose $\pi:(X, \phi) \overparen{\rightrightarrows}(Z, \rho)$ and $(Z, \rho)$ is equicontinuous. By $2.12,(X, \phi)$ is isomorphic to a subflow of $\left(Z^{T}, \hat{\rho}\right)$. Since $\left(Z^{T}, \hat{\rho}\right)$ is equicontinuous so is this subflow, and hence $(X, \phi)$ is equicontinuous. Since $(X, T)$ is type III (by 2.4) and since the orbit closure relation in an equicontinuous flow 
is an ICER, it follows that $(X, \phi)$ is minimal. Thus, $(Z, \rho)$ and the subflow of $\left(Z^{T}, \hat{\rho}\right)$ are minimal. By regularity of equicontinuous flows this implies $[1$, Theorem 3] that $(Z, \rho)$ and this subflow are isomorphic, and hence $(X, \phi)$ is isomorphic to $(Z, \rho)$. Now $(Z, \rho)$, as an infinite minimal equicontinuous flow, has a factor $(W, \delta)$ which is not isomorphic to it. But applying the above to $\pi_{1}:(X$, $\phi) \underset{\sim}{\rightarrow}(W, \delta)$, we obtain that $(X, \phi)$ is isomorphic to $(W, \delta)$. This contradiction proves the result.

One might describe Proposition 2.13 as implying that $(X, \phi)$ is "weakly mixing". Note that if $\phi$ is minimal, then since $X$ is metric, we do obtain the usual notion of weakly mixing, that is, $(X \times X, \phi \times \phi)$ is point transitive. One additional fact (which tends to support Conjecture $A$ ) is the lack of distal factors under the assumption of point transitivity.

Corollary 2.14. If $(X, T)$ is minimal prime, $\phi \in \operatorname{Aut}(X, T)$ with $(X, \phi)$ point transitive, then $(X, \phi)$ has no distal factors.

Proof. If $\pi:(X, \phi) \stackrel{\rightarrow}{\rightarrow}(Z, \rho)$ is distal, then by $2.12(X, \phi)$ is isomorphic to a subflow of $\left(Z^{T}, \hat{\rho}\right)$ and hence is distal. Since $(X, \phi)$ is transitive, then $(X, \phi)$ is minimal distal. However, the Furstenberg structure theorem than implies that $(X, \phi)$ has a nontrivial equicontinuous factor, contradicting 2.13 .

Based on this corollary and other examples, we make the following conjecture (stronger than Conjecture A).

Conjecture B. If $(X, T)$ is minimal prime and $\phi \in \operatorname{Aut}(X, T)$ with $(X$, $\phi)$ point transitive, then $(X, \phi)$ is minimal.

The proof of 2.13 also yields

Corollary 2.15. Let $(X, T)$ be minimal prime with $X$ infinite and $\phi \in$ $\operatorname{Aut}(X, T)$ with $(X, \phi)$ minimal. Then:

(1) If $(X, \phi) \stackrel{\Im}{\rightarrow}(Z, \rho)$ and $Z$ is nontrivial, then $\left(\operatorname{Reg}(Z), \rho_{0}\right) \stackrel{\rightarrow}{\rightarrow}(X, \phi)$ where $\left(\operatorname{Reg}(Z), \rho_{0}\right)$ is the regularizer of $(Z, \rho)[1],[2$, p. 108]

(2) $(X, \phi)$ has no proper nontrivial regular factors $(i . e .(X, \phi)$ is regular prime).

Proof. (1) By $2.12(X, \phi)$ is isomorphic to a minimal subflow $(M, \hat{\rho})$ of $\left(Z^{T}, \rho\right)$. Now if $\pi:\left(\operatorname{Reg}(Z), \rho_{0}\right) \simeq(Z, \rho)$ is the canonical map, then $\pi^{T}$ : $\left(\operatorname{Reg}(Z)^{T}, \hat{\rho}_{0}\right) \underset{\sim}{\longrightarrow}\left(Z^{T}, \hat{\rho}\right)$. Choose $\left(N, \hat{\rho}_{0}\right)$ minimal in $\left(\operatorname{Reg}(Z)^{T}, \hat{\rho}_{0}\right)$ with $\pi^{T}$ : $\left(N, \hat{\rho}_{0}\right) \underset{\sim}{\rightarrow}(M, \hat{\rho})$. By regularity $\left(N, \hat{\rho}_{0}\right) \simeq\left(\operatorname{Reg}\left(Z_{0}\right), \rho_{0}\right)$ and so $\left(\operatorname{Reg}\left(Z_{0}\right), \rho_{0}\right)$ $\simeq(X, \phi)$ as required.

(2) Suppose $\pi:(X, \phi) \Im(Z, \rho)$, with $Z$ nontrivial and $(Z, \rho)$ regular.

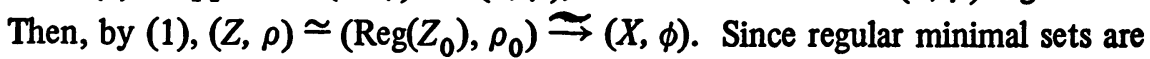
coalescent this means that $\pi$ is an isomorphism and so $(Z, \rho)$ is not a proper factor. 
Based on this we make the following conjecture:

Conjecture C. Let $(X, T)$ be minimal prime with $X$ infinite and $\phi \in$ $\operatorname{Aut}(X, T)$ with $(X, \phi)$ minimal. Then $(X, \phi)$ is prime.

In summary, the following classes of minimal prime flows arise, each of which contains the previous class: POD, purely prime, regular prime, prime. We know that purely prime does not imply POD; it is unknown if prime implies regular prime or regular prime implies purely prime. It is also unknown if a minimal flow of types II and III is purely prime.

In [5, Remark 3] the question arose as to whether a totally minimal flow having only graphs of powers as minimal sets in the product is prime. We now give an example to show that this is not the case. Let $(Y, \psi)$ be a POD flow as constructed in [5], and "split" another point not in the orbits of $x_{0}, \bar{x}_{0}, y_{0}, \bar{y}_{0}$ to obtain new points $\left(z_{0}, \bar{z}_{0}\right)$ and a new flow $(X, \rho)$. Then we have:

(a) $(X, \rho)$ is not prime, since $(Y, \psi)$ is a nontrivial factor.

(b) $\operatorname{Aut}(X, \rho)=\left\{\rho^{n}: n \in Z\right\}$, since $\phi \in \operatorname{Aut}(X, \rho)$ implies $\phi\left(z_{0}, \bar{z}_{0}\right) \in$ $O\left(z_{0}, \bar{z}_{0}\right) \cup O\left(\bar{z}_{0}, z_{0}\right)$ by double asymptoticity of $\left(z_{0}, \bar{z}_{0}\right)$.

(c) If $x \neq x_{1}, \partial\left(x, x_{1}\right) \supset \Gamma_{\rho^{n}}$ for some $n$; since this is true for $(Y, \psi)$ and for the pairs $\left(z_{0}, \bar{z}_{0}\right),\left(\bar{z}_{0}, z_{0}\right)$ we choose $n=0$.

Note that our example is a minimal almost 1-1 extension (there are points whose inverse image is a singleton) of POD flow. The next lemma shows that such flows will always produce examples of this type.

LEMMA 2.16. Let $(Y, T)$ be a minimal almost 1-1 extension of a POD transformation group $(X, T)$. Then $(Y, T)$ is totally minimal and has only graphs $\Gamma_{t}$ as minimal subsets in $(Y \times Y, T)$.

Proof. It is direct to verify that a minimal almost 1-1 extension of a weakly mixing transformation group is weakly mixing and hence totally minimal. Now let $y, y_{1} \in Y$. If $\rho:(Y, T) \widetilde{\longrightarrow}(X, T)$ and $\rho(y)=\rho\left(y_{1}\right)$, then $y$ and $y_{1}$ are proximal and $\bar{O}\left(y, y_{1}\right) \supset \Gamma_{e}$. If $\rho(y) \neq \rho\left(y_{1}\right)$ then $\rho \bar{O}\left(y, y_{1}\right) \supset \bar{O}\left(\rho(y), \rho\left(y_{1}\right)\right)$ $\supset \Gamma_{t}(X)$ for some $t(\neq e)$. If $\rho^{-1}\left(x_{0}\right)=\left\{y_{0}\right\}$ then $\left(y_{0}, y_{0} t\right) \in \bar{O}\left(y, y_{1}\right)$ and so $\bar{O}\left(y, y_{1}\right) \supset \Gamma_{t}$. This completes the proof.

Concerning the measure theoretic properties of prime flows, we have that if $(X, \psi)$ is POD, uniquely ergodic and totally ergodic, then for $n \neq 0\left(X, \psi^{n}\right)$ is purely prime and uniquely ergodic. Now $L$. Shapiro has proved the following (unpublished): given a fixed irrational $\alpha$, then for almost all $\beta$, the POD flow obtained in [5] is measure theoretically weak mixing (hence totally ergodic). On the other hand there exist purely prime flows which are not uniquely ergodic. This follows from an observation of Petersen [8, p. 229] that for some choices of $\alpha$ and $\beta$ the POD flow $(X, \psi)$ [5] has -1 as a measurable eigenvalue. So $\left(X, \psi^{2}\right)$ 
is purely prime and not uniquely ergodic. It is unknown whether every POD flow is uniquely ergodic.

3. "Number theoretic" properties of prime flows. We now consider analogues of number theoretic properties for purely prime and POD transformation groups. We first note that if $(X, T)$ is POD and $S_{1}$ and $S_{2}$ are isomorphic syndetic subgroups of $T$, then either $\left(X, S_{1}\right)$ is isomorphic to $\left(X, S_{1}\right)$ or $\left(X, S_{2}\right)$ is disjoint from $\left(X, S_{1}\right)$. This follows immediately from 2.6 since both $\left(X, S_{1}\right)$ and $\left(X, S_{2}\right)$ are purely prime. In the case of discrete POD flows we can be more specific, as the following generalisations of [5, Theorem 4.8] show.

Proposition 3.1. Let $(X, \psi)$ be POD. Then:

(1) If $m \neq 0 \neq n, m \neq \pm n$, then $\left(X, \psi^{m}\right)$ is disjoint from $\left(X, \psi^{n}\right)$.

(2) If $(X, \psi)$ is not isomorphic to $\left(X, \psi^{-1}\right)$, then $\left(X, \psi^{m}\right)$ is disjoint from $\left(X, \psi^{n}\right), 0 \neq m \neq n \neq 0$.

Proof. (1) Suppose $|n|>|m|$. We need only show there is no isomorphism $\pi:\left(X, \psi^{n}\right) \simeq\left(X, \psi^{m}\right)$. Let such a $\pi$ exist. Then since $\psi^{n}$ has an $n$th root it follows that $\psi^{m}$ has an $n$th root. Since $\operatorname{Aut}\left(X, \psi^{m}\right)=\left\{\psi^{l}: l \in Z\right\}(2.8)$, then $\psi^{j n}=\psi^{m}$ for some $j \neq 0$, and $j n=m$ which is a contradiction.

(2) We need only show the result for pairs $(m,-m) \neq(0,0)$. Suppose $\pi$ : $\left(X, \psi^{m}\right) \simeq\left(X, \psi^{-m}\right)$. Then the graph $\Gamma_{\pi}$ is minimal in $\left(X \times X, \psi^{m} \times \psi^{-m}\right)=$

$$
\left(X \times X, \psi^{m} \times \psi^{-m}\right)=\left(X \times X,\left(\psi \times \psi^{-1}\right)^{m}\right),
$$

and is also first category in $X \times X$. Now $\bigcup_{j=0}^{m-1}\left(\psi \times \psi^{-1}\right)^{j} \Gamma_{\pi}$ is closed and invariant in $\left(X \times X, \psi \times \psi^{-1}\right)$. Since $\left(X \times X, \psi \times \psi^{-1}\right)$ is minimal by assumption then $\bigcup_{j=0}^{m-1}\left(\psi \times \psi^{-1}\right)^{j} \Gamma_{\pi}=X \times X$, which is a contradiction.

In general, we cannot show that for any POD flow, $(X, \psi)$ is not isomorphic to $\left(X, \psi^{-1}\right)$. However we conjecture that this is the case for the particular class of POD flows discussed in [5].

We now go on to discuss properties of purely prime flows which are reminiscent of the properties of prime numbers.

LEMma 3.2. Let $\left(X_{i}, T\right), i=1, \ldots, n$, be purely prime flows such that $\left(X_{1} \times \cdots \times X_{n}, T\right)=(\bar{X}, T)$ is minimal. Then

1. The only minimal subsets of $(\bar{X} \times \bar{X}, T)$ are the graphs $\Gamma_{\phi_{1}} \times \cdots \times \phi_{n}=$ $\Gamma_{\phi_{1}} \times \cdots \times \Gamma_{\phi_{n}}$ where $\phi_{i} \in \operatorname{Aut}\left(X_{i}, T\right)$.

2. $\operatorname{Aut}\left(X_{1} \times \cdots \times X_{n}, T\right)=\operatorname{Aut}\left(X_{1}, T\right) \times \cdots \times \operatorname{Aut}\left(X_{n}, T\right)$.

3. If $x, y \in \bar{X}$ with $x \neq y$ the $\bar{O}(x, y) \supset \Gamma_{\phi}, \phi \neq \mathrm{id}, \phi \in \operatorname{Aut}(\bar{X}, T)$.

Proof. We first consider the case $n=2$. Let $M$ be a minimal subset of $(\bar{X} \times \bar{X}, T)$. Considering the natural projections $\mathrm{pr}_{1}: \bar{X} \times \bar{X} \rightarrow X_{1} \times X_{1}, \mathrm{pr}_{2}$ : $\bar{X} \times \bar{X} \rightarrow X_{2} \times X_{2}$, then $\operatorname{pr}_{1} M$ is minimal in $X_{1} \times X_{1}$ and $\operatorname{pr}_{2} M$ is minimal in $X_{2} \times X_{2}$. Since the flows $\left(X_{1}, T\right)$ and $\left(X_{2}, T\right)$ are type II, then $\operatorname{pr}_{1} M=$ 
$\Gamma_{\phi_{1}}$ and $\operatorname{pr}_{2} M=\Gamma_{\phi_{2}}$ for some $\phi_{i} \in \operatorname{Aut}\left(X_{1}, T\right), i=1,2$. So $M \subset \Gamma_{\phi_{1}} \times \Gamma_{\phi_{2}}$ $=\Gamma_{\phi_{1} \times \phi_{2}}$. Since $(\bar{X}, T)$ is minimal so is $\Gamma_{\phi_{1} \times \phi_{2}}$ and thus $M=\Gamma_{\phi_{1} \times \phi_{2}}$, proving (1). For (2), let $\pi \in \operatorname{Aut}(\bar{X}, T)$. Then $\Gamma_{\pi}$ is minimal in $(\bar{X} \times \bar{X}, T)$ and so $\Gamma_{\pi}=\Gamma_{\phi_{1} \times \phi_{2}}$. Hence $\pi=\phi_{1} \times \phi_{2}$ and we obtain the result. For (3), let $x$, $y \in \bar{X}, x \neq y$ and suppose $\Delta_{\bar{X}}$ is the only minimal subset of $\bar{O}(x, y)$. Then $\Delta_{X_{1}}$ is the only minimal subset of $\operatorname{pr}_{1} \bar{O}(x, y)=\bar{O}\left(x_{1}, y_{1}\right)$ and $\Delta_{X_{2}}$ is the only minimal subset of $\mathrm{pr}_{2} \bar{O}(x, y)=\bar{O}\left(x_{2}, y_{2}\right)\left[x=\left(x_{1}, x_{2}\right), y=\left(y_{1}, y_{2}\right)\right]$. But, without loss of generality, we may assume $x_{1} \neq y_{1}$ and hence $\bar{O}\left(x_{1}, y_{1}\right) \supset \Gamma_{\phi_{1}}$ for some id $\neq \phi_{1} \in \operatorname{Aut}\left(X_{1}, T\right)$. This contradiction implies that $\bar{O}(x, y) \supset M$ with $M$ minimal and $M \neq \Delta \bar{X}$. Hence, by (2), $M=\Gamma_{\phi_{1}} \times \phi_{2}$ with $\phi_{1} \times \phi_{2} \neq$ id.

For general $n$ we obtain the results by a straightforward induction argument modelled on the above.

The first result we obtain is reminiscent of the Unique Factorisation theorem in number theory.

Proposition 3.3. Let $\left(X_{i}, T\right), i=1, \ldots, n$, by purely prime flows, each $X_{i}$ metric, and with nontrivial automorphisms inducing minimal discrete flows. Suppose $\left(X_{1} \times \cdots \times X_{n}, T\right)$ is minimal. Then the only factors of $\left(X_{1} \times \cdots \times\right.$ $\left.T_{n}, T\right)=(\bar{X}, T)$ are $\left(X_{j_{1}} \times \cdots \times X_{j_{s}}, T\right), 1 \leqslant j_{1}<\cdots<j_{s} \leqslant n$.

Proof. We use induction on $n$. The result is trivially true for $n=1$. We assume the result for $1,2, \ldots, n-1$.

Let $R$ be an ICER on $(\bar{X}, T)$ with $R \neq \Delta_{\bar{X}}$. Then by Lemma 3.2, $R \supset \Gamma_{\phi}^{\text {L }}$ with $\bar{\phi}=\phi_{1} \times \cdots \times \phi_{n} \neq \mathrm{id}, \bar{\phi} \in \operatorname{Aut}(\bar{X}, T)$. Now for each $\phi_{i} \neq \mathrm{id},\left(X_{i}, \phi_{i}\right)$ is minimal and weakly mixing (Proposition 2.13). Let $\phi_{j_{k}}=\mathrm{id}_{j_{k}}$ with $1 \leqslant j_{1}<$ $\cdots<j_{s} \leqslant n(s<n)$, and consider $(Z, \widetilde{\phi})=\left(X_{k_{1}} \times \cdots \times X_{k_{n-s}}, \phi_{k_{1}} \times \cdots \times\right.$ $\left.\phi_{k_{n-s}}\right)$, where $k_{m} \neq j_{l}$ for any $m, l$. It follows by [7, Theorem 16(2)] that (Z, $\widetilde{\phi}$ ) is weak mixing (one can also easily give a direct proof of this). Pick $z \in Z$ with a dense $\tilde{\phi}$-orbit. Fix $w \in X_{j_{1}} \times \cdots \times X_{j_{s}}$. Regarding $(w, z) \in \bar{X}$, then $\bar{\phi}(w, z)=(w, \widetilde{\phi}(z))$ and so $\left((w, z),\left(w, \tilde{\phi}^{r}(z)\right)\right) \in R$ for every $r$. By density of orbit of $z$ we have $(w, y) \in R$ for every $y \in Z$. Hence $(X / R, T)$ is a factor of $\left(X_{j_{1}} \times \cdots \times X_{j_{s}}, T\right)$. Since $s<n$ we now use the induction hypothesis to complete the proof.

Note that if $T$ is $\sigma$-compact the metric assumption follows by Proposition 2.11 .

We now study the assumption of minimality of the $n$-fold product in Proposition 3.3. Note that in general if we have 3 transformation groups which are pairwise disjoint then minimality of the 3-fold product does not necessarily follow: For example, let $\alpha$ and $\beta$ be rationally independent irrationals in $[0,1)$ and consider the rotations on $[0,1)$ through $\alpha, \beta$ and $\alpha+\beta$. These three flows are 
pairwise disjoint but the product of any two has the third as a factor showing the nonminimality of the three-fold product. However, in purely prime flows with minimal automorphisms, pairwise disjointness does yield minimality of larger products.

Proposition 3.4. Let $\left(X_{i}, T\right), i=1, \ldots, n$, be purely prime flows, each $X_{i}$ metric, and with nontrivial automorphisms inducing minimal discrete flows. Suppose that $\left(X_{i} \times X_{j}, T\right)$ is minimal for $i \neq J$. Then $\left(X_{1} \times \cdots \times X_{n}, T\right)$ is minimal.

Proof. By induction on $n$. The result is trivially true for $n=2$. Suppose we have the result for $n-1$. Then $\left(X_{1} \times \cdots \times X_{n-1}, T\right)$ is minimal, and to complete the proof we have to show that $\left(X_{n}, T\right)$ is not a factor of $\left(X_{1} \times \cdots \times\right.$ $\left.X_{n-1}, T\right)$ (Proposition 2.6). Now if $\left(X_{n}, T\right)$ is a factor of $\left(X_{1} \times \cdots \times X_{n-1}\right.$, $T)$ then for some $j, 1 \leqslant j \leqslant n-1$, we must have $\left(X_{n}, T\right)$ is isomorphic to $\left(X_{j}, T\right)$ (using Proposition 3.3 and primeness of $\left.\left(X_{n}, T\right)\right)$. However, by assumption $\left(X_{j} \times\right.$ $\left.X_{n}, T\right)$ is minimal. So this is impossible and the proposition is proved.

We first apply these results to discrete POD flows.

COROllary 3.5. Let $(X, \phi)$ be POD and consider integers $k_{i} \neq 0, i=1$, $\ldots, n$, with $k_{i} \neq \pm k_{j}$ for $i \neq j$. Then

(1) $\left(X \times \cdots \times X, \phi^{k_{1}} \times \cdots \times \phi^{k_{n}}\right)$ is minimal.

(2) The only factors of the flow in (1) are the obvious direct factors.

If, in addition, $(X, \phi)$ is not isomorphic to $\left(X, \phi^{-1}\right)$ then (1) and (2) hold for any $k_{i} \neq 0, i=1, \ldots, n$, with $k_{i} \neq k_{j}$ for $i \neq j$.

Proof. This follows immediately by Propositions 3.1, 3.3 and 3.4.

COROLlARY 3.6. Let $(X, \phi)$ be POD and consider any sequence of integers $\left(k_{n}\right), k_{n} \neq 0$, with $k_{n} \neq \pm k_{m}$ for $n \neq m$. Then $\left(\Pi_{n=1}^{\infty} X, \Pi_{n=1}^{\infty} \phi^{k_{n}}\right)$ is minimal. If, in addition, $(X, \phi)$ is not isomorphic to $\left(X, \phi^{-1}\right)$, then we need only assume $k_{n} \neq k_{m}$.

PROOF. $\left(\Pi_{n=1}^{\infty} X, \Pi_{n=1}^{\infty} \phi^{k} n\right)$ is the inverse limit of the minimal system $\left\{\left(\Pi_{m=1}^{n} X, \Pi_{m=1}^{n} \phi^{k} m\right) \mid n \geqslant 1\right\}$ and hence is minimal.

Note that one can similarly prove that if $\left(X_{i}, T\right), i \geqslant 1$, are purely prime with minimal nontrivial automorphisms and if $\left(X_{i} \times X_{j}, T\right)$ is minimal for $1 \neq j$, then $\left(\Pi_{i=1}^{\infty} X_{i}, T\right)$ is minimal.

We close with some observations in the case of a real POD flow $\left(X,\left\{S_{t}\right\}\right)$. If $0 \neq t_{1} \neq t_{2} \neq 0$ then, by Proposition 2.6, either $\left(X, S_{t_{1}}\right)$ is isomorphic to $\left(X, S_{t_{2}}\right)$ or $\left(X \times X, S_{t_{1}} \times S_{t_{2}}\right)$ is minimal. We are unable, in general, to determine which case occurs. Suppose that $\phi:\left(X, S_{t_{1}}\right) \simeq\left(X, S_{t_{2}}\right)$. Then for $t \in R$ we have 


$$
\left(\phi S_{t} \phi^{-1}\right) S_{t_{2}}=\phi S_{t} S_{t_{1}} \phi^{-1}=\phi S_{t_{1}} S_{t} \phi^{-1}=S_{t_{2}}\left(\phi S_{t} \phi^{-1}\right)
$$

Therefore $\phi S_{t} \phi^{-1} \in \operatorname{Aut}\left(X, S_{t_{2}}\right)=\left\{S_{r} \mid r \in R\right\}$. Hence $\phi S_{t} \phi^{-1}=S_{\eta(t)}$ for some function $\eta: R \rightarrow R$. By the total minimality of $\left(X,\left\{S_{t}\right\}\right)$ we can see that $\eta$ is one-to-one and so if there is one isomorphic pair then there are uncountably many isomorphic pairs. It is also easy to show that $\eta(t+s)=\eta(t)+\eta(s)$ for $t, s \in R$ and $\eta\left(r t_{1}\right)=r t_{2}$ for rational $r$. If $\eta$ were continuous then being an additive group homomorphism of $R$ it would take the form $\eta(t)=k t$ for some $k \in R$ and then $\phi$ would be an isomorphism between the flows $\left(X,\left\{S_{t}\right\}\right)$ and $\left(X,\left\{S_{k t}\right\}\right)$. In general we cannot prove continuity of $\eta$, but under a certain condition, which holds in our example of $\S 1$, we can prove continuity.

Lemma 3.7. Suppose $\left(X,\left\{S_{t}\right\}\right)$ has a pair of asymptotic points. Then $\eta$ is continuous.

Proof. Since $\eta$ is an additive group homomorphism we need only show continuity at 0 . If $\eta$ is not continuous at 0 , there exists $\epsilon>0$ and a sequence $t_{n} \rightarrow 0$ with $\left|\eta\left(t_{n}\right)\right|>\epsilon$. Since $S_{t_{n}} \rightarrow$ id, $S_{\eta\left(t_{n}\right)} x=\phi S_{t_{n}} \phi^{-1} x \rightarrow x(x \in X)$. Now if the sequence $\eta\left(t_{n}\right)$ is bounded then we can choose a cluster point $\alpha$ and we would get $S_{\alpha} x=x(x \in X)$ and $|\alpha| \geqslant \epsilon$. This contradicts minimality of $(X$, $\left.S_{\alpha}\right)$. So $\left(\eta\left(t_{n}\right)\right)$ is unbounded. Since $\eta(-t)=-\eta(t)$ we also get $S_{\eta\left(-t_{n}\right)} x \rightarrow x$ and $\left(\eta\left(-t_{n}\right)\right)$ is unbounded. So we can choose subsequences, $\left(s_{n}\right),\left(u_{n}\right)$ with one a subsequence of $\left(t_{n}\right)$ and the other from $\left(-t_{n}\right)$ with $\eta\left(s_{n}\right) \rightarrow+\infty, \eta\left(u_{r}\right) \rightarrow-\infty$. Now pick $(x, y)$ asymptotic with $x \neq y$. Then $S_{\eta\left(s_{n}\right)}(x, y) \rightarrow(x, y)$ and $S_{\eta\left(u_{n}\right)}(x, y) \rightarrow(x, y)$ which contradicts asymptoticity. Therefore $\eta$ is continuous.

In the case when $\eta$ is continuous, and therefore of the form $\eta(t)=k t$, it would be interesting to know what values $k$ can take. One would expect the values \pm 1 , are the only possibilities.

\section{REFERENCES}

1. J. Auslander, Regular minimal sets. I, Trans. Amer. Math. Soc. 123 (1966), 469479. MR 33 \#1845.

2. R. Ellis, Lectures on topological dynamics, Benjamin, New York, 1969. MR 42 \#2463.

3. R. Ellis, S. Glasner and L. Shapiro, P. I. flows, Advances in Math. 17 (1975), 213261.

4. R. Ellis and H. B. Keynes, A characterization of the equicontinuous structure relation, Trans. Amer. Math. Soc. 161 (1971), 171-183. MR 43 \#8069.

5. H. Furstenberg, H. B. Keynes and L. Shapiro, Prime flows in topological dynamics, Israel J. Math. 14 (1973), 26-38. MR 47 \#9588.

6. H. B. Keynes, The structure of weakly mixing transformation groups, Illinois J. Math. 15 (1971), 475-489. MR 44 \#3306.

7. R. Peleg, Weak disjointness of transformation groups, Proc. Amer. Math. Soc. 33 (1972), 165-170. MR 45 \#7694. 
8. K. Petersen, Spectra of induced transformations, Recent Advances in Topological Dynamics, Lecture Notes in Math., vol. 318, Springer-Verlag, Berlin and New York, 1973, pp. 226-230.

9. K. Petersen and L. Shapiro, Induced flows, Trans. Amer. Math. Soc. 177 (1973), 375-390. MR 48 \#1200.

SCHOOL OF MATHEMATICS, UNIVERSITY OF MINNESOTA, MINNEAPOLIS, MINNESOTA 55455

SCHOOL OF MATHEMATICAL AND PHYSICAL SCIENCES, UNIVERSITY OF SUSSEX, BRIGHTON, ENGLAND 